\title{
Preparation and Application of Film Sensor for Metal Structure Crack Monitoring
}

\author{
Bo HOU, Yuting HE** Ronghong CUI, Teng ZHANG \\ Engineering College, Air Force Engineering University, Xi'an, 710038, China \\ cross $^{\text {ref }}$ http://dx.doi.org/10.5755/j01.ms.21.4.9623
}

Received 27 January 2015; accepted 11 September 2015

\begin{abstract}
A crack monitoring technique is desired to ensure the safety and reliability of metallic structures. In the present study, a conductive film sensor was presented to monitor structural cracks in metal structures in real-time based on the electrical potential method. First, a Ti/TiN film sensor was prepared on the fatigue critical portion of a 2A12-T4 aluminum alloy specimen by vacuum ion plating technology, which allows firm integration with the metal surface. A finite element model (FEM) of the Ti/TiN film sensor was then constructed and the changes in the output of the sensor along with corresponding changes in crack propagation were discussed. The results indicated that the Ti/TiN film sensor has high sensitivity to cracks and it is feasible to monitor structural surface cracks using the sensor. Finally, crack monitoring experiments were carried out based on the Ti/TiN film sensor. Experimental results showed that the output potential curve of the Ti/TiN film sensor contained several regions, which corresponded to plastic deformation accumulation, crack propagation, and sensor failure, respectively. Therefore, the information on the origination and propagation of structural cracks can be gained through analyzing changes in slope of the output potential values of the Ti/TiN film sensor with respect to time.

Keywords: crack monitoring, Ti/TiN film, electrical potential method, vacuum ion plating, metal structure.
\end{abstract}

\section{INTRODUCTION}

Metal is the material most commonly used for loadcarrying structures. These structures are subjected to high alternating stresses and impacts during their use, which may cause fatigue crack growth and, ultimately, rupture failure. Monitoring of fatigue crack damage may be the most effective way to ensure service safety of metal structures.

Several crack monitoring methods have recently been presented [1-5]. However, in terms of precision, reliability, economy, and durability, these techniques have limitations $[6,7]$. Therefore, it is increasingly urgent to develop an effective structural crack monitoring technique. In recent years, the electrical potential method has been given a great deal of attention in the field of nondestructive testing and this may be due to its many advantages, particularly the fact that it is based on a simple principle. However, there are still certain issues in monitoring crack by using electrical potential technique directly [8]. Therefore, a new method, preparing conductive film sensors on the surface of substrate and measuring the changes in electrical information of sensor, was developed for monitoring crack damage.

Most of film sensors for metal structure crack monitoring in existing literatures [9-13] were combined with substrate by various adhesives. This combination method may hinder structural crack from accessing to film sensors and may make the propagation direction of crack in film sensor different from that in substrate [13]. Yet the $\mathrm{Ti} / \mathrm{TiN}$ film sensor in this paper was different. The Ti/TiN film sensor was plated on the fatigue critical portion of

\footnotetext{
${ }^{*}$ Corresponding author. Tel.: +86-29-84787082, fax: +86-29-84787082

E-mail address: hyt666@tom.com (Y.He)
}

substrate by ion plating technology. In this way, the Ti/TiN film sensor had a good bonding strength and an excellent damage consistency with substrate.

In this paper, a suitable Ti/TiN conductive film sensor with a specific shape was prepared on a metal structural surface through a vacuum ion plating technique. The output characteristics of the Ti/TiN film sensor were studied through finite element simulation and analysis and structural crack monitoring experiments were carried out to verify the feasibility of monitoring crack growth with the Ti/TiN film sensor.

\section{SENSOR PREPARATION}

\subsection{Materials}

A 2A12-T4 aluminum alloy specimen with a center bore was used in this study. The composition of the

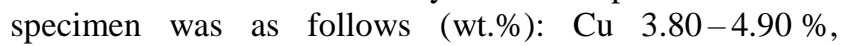
$\mathrm{Mg} 1.20-1.80 \%, \mathrm{Si} \leq 0.50 \%, \mathrm{Fe} \leq 0.50 \%, \mathrm{Zn} \leq 0.30 \%$, $\mathrm{Ni} \leq 0.10 \%$, impurity $\leq 0.10 \%$, and $\mathrm{Al}$ balance. The shape and dimension of the specimen are shown in Fig. 1.

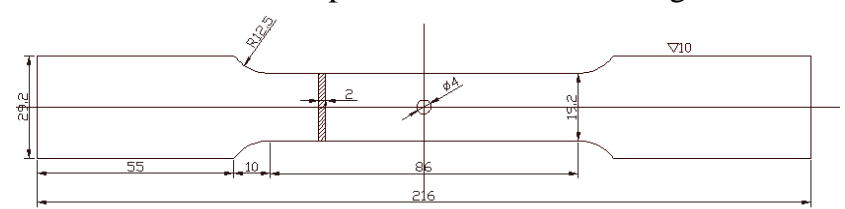

Fig. 1. Shape and dimensions of the specimen (units: $\mathrm{mm}$ )

\subsection{Specimen pretreatment}

Since the material of the specimen was 2A12-T4 aluminum alloy, the anodic oxidation process was used to space out the substrate and the Ti/TiN conductive film. The main process parameters are shown as Table 1 . 
Table 1. Process parameters for anodic oxidation

\begin{tabular}{|c|c|}
\hline Process conditions & Parameters \\
\hline $\mathrm{H}_{2} \mathrm{SO}_{4}[\mathrm{~g} / \mathrm{L}]$ & $40-50$ \\
\hline $\mathrm{H}_{3} \mathrm{BO}_{3}[\mathrm{~g} / \mathrm{L}]$ & $5-10$ \\
\hline $\mathrm{Al}^{3+}[\mathrm{g} / \mathrm{L}]$ & $<15$ \\
\hline Temperature, $^{\circ} \mathrm{C}$ & $25-30$ \\
\hline DC Voltage, $\mathrm{V}$ & $10-20$ \\
\hline Current Density, $\mathrm{A} / \mathrm{dm}^{2}$ & $0.4-2.5$ \\
\hline Time Horizon, $\mathrm{min}$ & $30-60$ \\
\hline
\end{tabular}

\subsection{Ti/TiN film preparation}

Hollow cathode discharge deposition technology [14] was applied in this study to deposit the conductive film and $\mathrm{Ti} / \mathrm{TiN}$ was used as the material for the conductive film.

After mechanical polishing, the specimens were ultrasonically cleaned for approximately $5 \mathrm{~min}$ by alternating between pure water and acetone. Specimens were then set on a holder and argon ion bombardment was performed at the bias voltage of $500 \mathrm{~V}$ in a vacuum of $2 \mathrm{~Pa}$ for approximately $5 \mathrm{~min}$ to improve the binding force between the film and the specimen. Finally, the Ti/TiN film was deposited with IPB30/30 ion plating equipment. Table 2 shows the deposition parameters of the vacuum ion plating technology.

The deposited $\mathrm{Ti} / \mathrm{TiN}$ film consists of two layers: the Ti film layer and the TiN film layer. As the sensing function layer at the top of the composite film, the TiN film is used to monitor structural crack. As the transition layer between substrate and the TiN film, the Ti film can improve the bonding strength of the film-substrate interface. The thickness of Ti/TiN film was controlled by depositing parameters, mainly depositing time.

The specimen with the Ti/TiN film sensor is shown in Fig. 2. It may be observed in Fig. 2 that the ring part of the $\mathrm{Ti} / \mathrm{TiN}$ film sensor is $0.5 \mathrm{~mm}$ wide, and the lead wire is $1 \mathrm{~mm}$ wide.

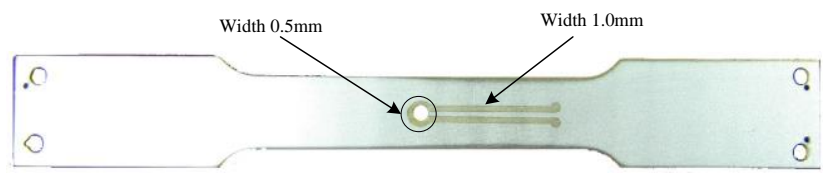

Fig. 2. Ti/TiN film sensor obtained on the specimen

\subsection{Characterization of Ti/TiN film}

The hypothetical sensing mechanism of the Ti/TiN film sensor is as follow. If the Ti/TiN film sensor is integrated with substrate firmly, the interaction at the interface would cause consistent damage to the film when damage occurs in substrate; that is to say, the Ti/TiN film sensor develops crack in correspondence and synchronously as structural crack initiates and gradually propagates over it. As a crack propagates through the substrate as well as the Ti/TiN film sensor, due to a continuous reduction in conductive area of the Ti/TiN film, there will be obvious variations of potential field, which will be shown as fluctuation of electrical potential values. Consequently, the information about structural crack can be obtained through monitoring the changes in electrical potential values of the $\mathrm{Ti} / \mathrm{TiN}$ film sensor.

It follows from the sensing mechanism that a reliable Ti/TiN film sensor should be able to maintain a steady output of electrical potential difference at constant current before application of load.

The electrical potential difference cross the Ti/TiN film sensor was measured. The result of measurement showed that the output of electrical potential difference (or resistance) across $\mathrm{Ti} / \mathrm{TiN}$ film sensor was very stable at a constant DC current before application of load.

\section{FINITE ELEMENT SIMULATION}

A finite element model (FEM) of the Ti/TiN film sensor was constructed to verify the feasibility of monitoring cracks using this technique. Cracks propagating from $0 \mathrm{~mm}$ to $0.4 \mathrm{~mm}$ in length were simulated using FEM.

\subsection{Finite element model of the sensor}

The geometric model of the Ti/TiN film sensor is shown in Fig. 3. The outside diameter of the sensor was $5 \mathrm{~mm}$ and the inside diameter was $4 \mathrm{~mm}$. There were symmetrical cracks on both sides of the model.

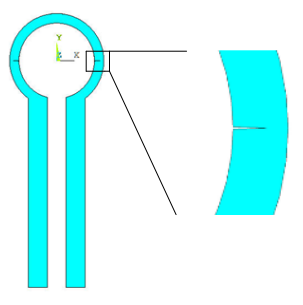

Fig. 3. Geometric model of the sensor

Since the thickness of the Ti/TiN film sensor is only several microns, a two-dimensional FEM was constructed to study the output characteristics of the Ti/TiN film sensor. As the preparation technique of the Ti/TiN film sensor was HCD, the sensor was considered to be an isotropic material in this study and the resistivity was defined as $0.089 \Omega \cdot \mathrm{m}$. PLANE230 elements were used to mesh the whole model. This is an electric element type which is 2-D and contains 8 nodes. As shown in Fig. 4, the model was meshed and the crack-tip elements were refined to ensure precision in the calculations.

Table 2. Process parameters for the deposition of the conductive film

\begin{tabular}{|c|c|c|c|c|c|}
\hline Bombardment cleaning & Parameters & Ti Deposition & Parameters & TiN Deposition & Parameters \\
\hline Bombardment Bias, $\mathrm{V}$ & $550-600$ & Substrate bias, $\mathrm{V}$ & $200-250$ & Substrate bias, $\mathrm{V}$ & $150-200$ \\
\hline Ar partial pressure, $\mathrm{Pa}$ & 0.4 & Beam current, $\mathrm{A}$ & $40-60$ & Beam current, $\mathrm{A}$ & $50-80$ \\
\hline Time, min & $5-10$ & Time, min & $20-30$ & Time, min & $10-60$ \\
\hline Temperature, ${ }^{\circ} \mathrm{C}$ & 200 & Temperature, ${ }^{\circ} \mathrm{C}$ & 200 & $\mathrm{~N}_{2}$ partial pressure, $\mathrm{Pa}$ & 0.133 \\
\hline
\end{tabular}




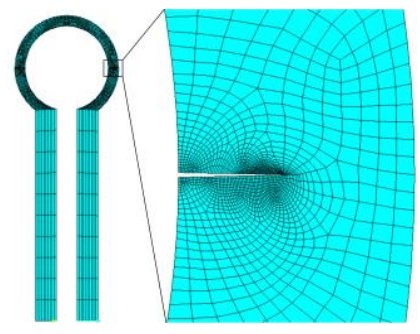

Fig. 4. Finite element model of the Ti/TiN film sensor

The point $\mathrm{D}$ was set to be the input point of the $1 \mathrm{~A} \mathrm{DC}$ current, the point $\mathrm{C}$ was set to be the output point of the DC current, and the point $\mathrm{C}$ was defined as a point of zero electric potential. A steady state analysis was performed. Finally, the electric potential at the point $\mathrm{D}$ was acquired in the post-processing stage.

The point $\mathrm{D}$ was set to be the input point of the $1 \mathrm{~A} \mathrm{DC}$ current, the point $\mathrm{C}$ was set to be the output point of the $\mathrm{DC}$ current, and the point $\mathrm{C}$ was defined as a point of zero electric potential. A steady state analysis was performed. Finally, the electric potential at the point D was acquired in the post-processing stage.

\subsection{Output characteristics of the sensor}

The distributions of electric potential were acquired in post-processing through simulation analyses when the crack length was $0 \mathrm{~mm}$ and $0.4 \mathrm{~mm}$ respectively, which is shown in Fig. 5.
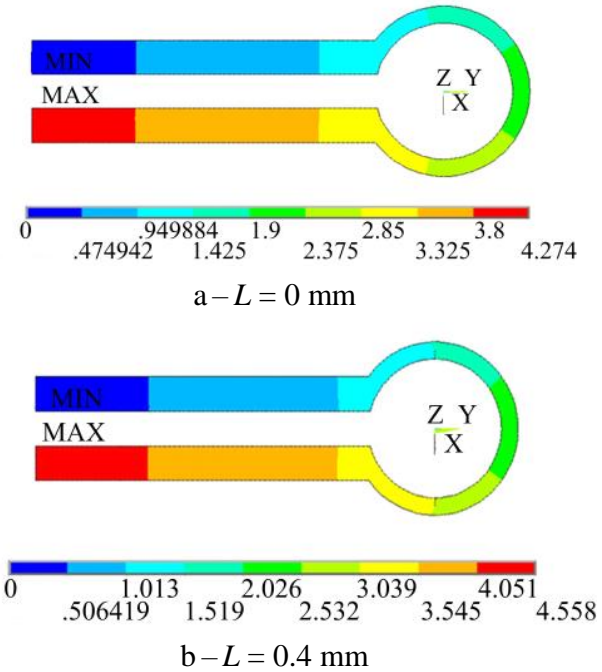

Fig. 5. Potential distribution maps of the sensor with different crack lengths

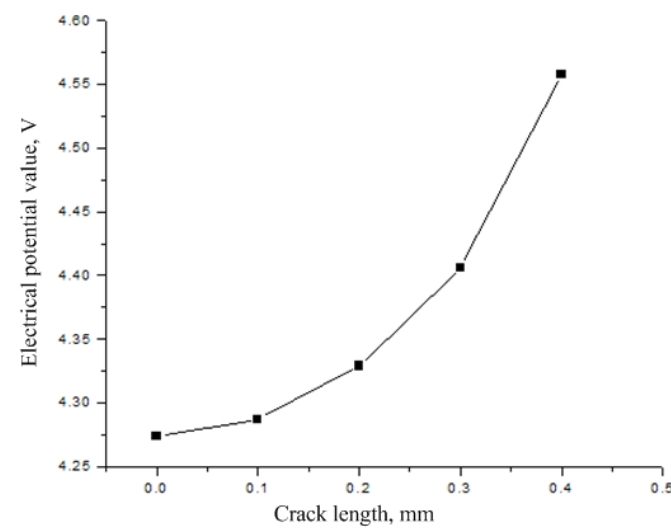

Fig. 6. Variation of the electric potential difference along with crack propagation
It may be observed that the distributions of electric potentials are altered at the crack-tip. The output characteristics of the sensor with crack lengths of $0,0.1$, $0.2,0.3$, and $0.4 \mathrm{~mm}$ were determined by the FEM. The electric potential changes of the point $\mathrm{D}$ with crack propagation are shown in Fig. 6. It may be observed in Fig. 6 that the potential at point D increases gradually along with the increase of the crack length. Therefore, the sensor can be used to effectively monitor crack origination and propagation.

\section{CRACK MONITORING EXPERIMENT}

A crack monitoring experiment was carried out with the Ti/TiN film sensor and correlations between the output values of the Ti/TiN film sensor and the length of the fatigue crack were studied in this experiment. A schematic illustration of the crack monitoring circuit is shown in Fig. 7, in which $\mathrm{R}$ is a symbol of the resistance of the $\mathrm{Ti} / \mathrm{TiN}$ film sensor, and the constant voltage power with the slide rheostat can be considered as a constant direct current power. During the experiment, the ART USB2828 data acquisition card and the VICTOR86B digital multimeter were used to record the output data of the Ti/TiN film sensor along with the crack propagation. An optical microscope (resolution: $0.1 \mathrm{~mm}$ ) was used to observe the crack length.

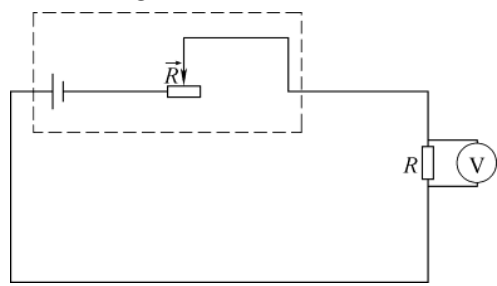

Fig. 7. Schematic of the crack monitoring circuit

\subsection{Experimental details}

The scene of the crack monitoring experiment is shown in Fig. 8. EHF-EA5 materials testing system (load error less than $1 \%$ ) was used to load specimens in air.

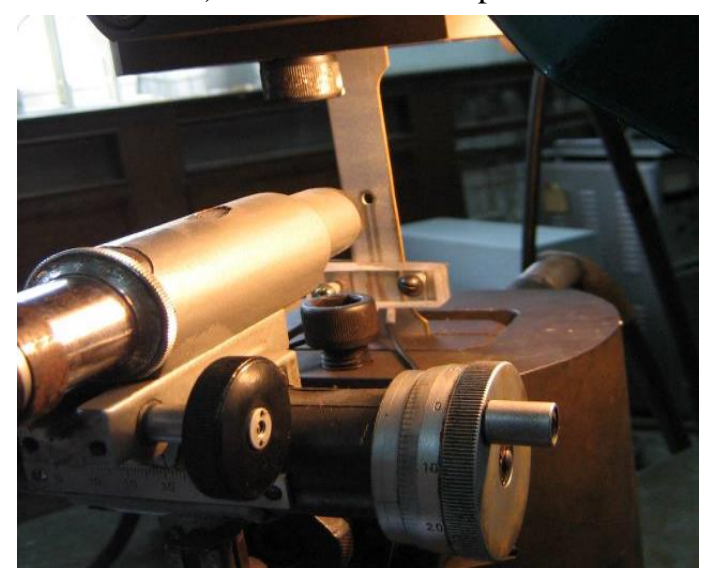

Fig. 8. Scene of the crack monitoring experiment

The experiment was carried out under constant amplitude loading with the following parameters of cyclic loading: stress ratio $R=0.05$ and loading frequency $f=10 \mathrm{~Hz}$. In order to perform a full observation of crack propagation and in order to shorten the experimental time, the maximum stress was set to different values at different 
experimental stages. First, the maximum stress was $60 \mathrm{MPa}$ at the beginning of the experiment. The maximum stress was then reduced to $50 \mathrm{MPa}$ after the initiation of plastic deformation. Finally, the maximum stress was reduced to $40 \mathrm{MPa}$ after a structural fatigue crack was observed.

\subsection{Results and discussion}

The morphology of the Ti/TiN film sensor after the fatigue crack monitoring experiment is shown in Fig. 9. It may be observed in Fig. 9 that there was no phenomenon of discontinuity or desquamation on the fractured Ti/TiN film sensor. This indicates that the Ti/TiN film sensor was integrated with the 2A12-T4 substrate firmly during the whole experiment and the Ti/TiN film sensor exhibited good damage consistency with the substrate, which is the precondition and basis of monitoring structural crack by this method [8].

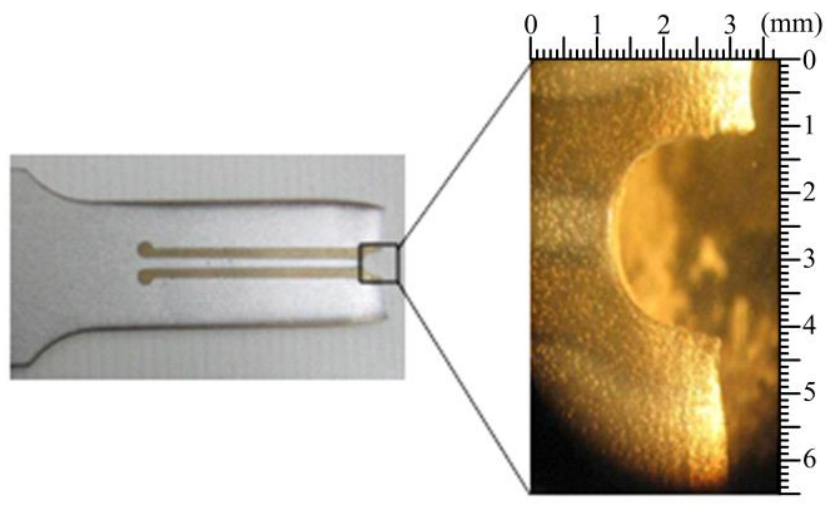

Fig. 9. Morphology of the Ti/TiN sensor after fracture

The changes in the output values of the Ti/TiN film sensor along with the experiment time course is shown in Fig. 10. It may be observed in Fig. 10 that the output values of the $\mathrm{Ti} / \mathrm{TiN}$ film sensor were proportional to the development process of the fatigue crack damage.

At the beginning of the curve in Fig. 10, the electric potential increases linearly before point A. It is likely the result of the resistance strain effect of the Ti/TiN film sensor and corresponds to the result of the [13]. In the next part of this curve, there are two regions where the electric potential increases rapidly, which show good agreement with the results of [7] and [15]. The first starts at point A and the second starts at point $\mathrm{C}$. The first rapid rise of the electric potential may correspond to plastic deformation accumulation of the specimen because the core hole of the specimen became oval at point B during the crack monitoring experiment. The second rapid rise of the potential values may correspond to the resistance increase of the sensor along with the crack origination and propagation because the crack origination was observed at point $\mathrm{C}$ with the optical microscope during the crack monitoring experiment. In the last part of the curve, the potential values of the sensor suddenly decrease. This sudden decrease may correspond to a gradual failure of the connection between the film sensor and aluminum alloy substrates because a cross-running crack was observed on the Ti/TiN film sensor at that time. The total change trend of the Ti/TiN film sensor output during the process of crack monitoring is very similar to that shown in the [10] and [15].

It can be concluded that information on the structural crack origination and propagation can be obtained through studying the output characteristic of the Ti/TiN film sensor and it is feasible to monitor cracks in metal structures based on the electrical potential method with a Ti/TiN film sensor.

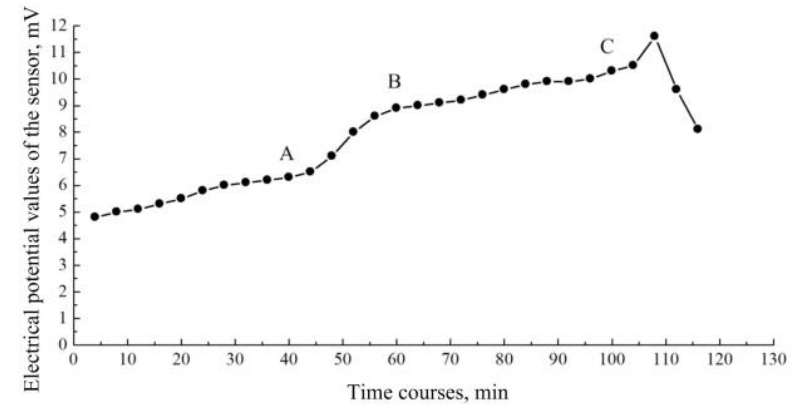

Fig. 10. Variation of the potential values of the Ti/TiN film sensor along with time

\section{CONCLUSIONS}

1. A Ti/TiN film sensor was prepared on the fatigue critical part of a 2A12-T4 aluminum alloy specimen by ion plating technology and the sensor firmly integrated with the substrate.

2. A FEM of the Ti/TiN film sensor was constructed and the FEM simulation confirmed that the Ti/TiN film sensor is sensitive to the growth of a crack and that it is feasible to monitor the surface crack using the sensor.

3. Results of a crack monitoring experiment showed that structural crack origination and propagation can be monitored by analyzing changes in slope of the output values of the sensor with respect to time.

\section{Acknowledgments}

The authors thank the anonymous reviewers for their critical and constructive review of the manuscript. This study was co-supported by the National Natural Science Foundation of China (No. 51201182).

\section{REFERENCES}

1. Hongo, A., Kojima, S., Komatsuzaki, S. Application of Fiber Bragg Grating Sensors and High-Speed Interrogation Techniques Structural Control and Health Monitoring $12(3-4)$ 2005: pp. 269-282.

2. Roach, D. Real Time Crack Detection Using Mountable Comparative Vacuum Monitoring Sensors Smart Structures Systems 5 (4) 2009: pp. 317-328. http://dx.doi.org/10.12989/sss.2009.5.4.317

3. Rabiei, M., Modarres, M. Quantitative Methods For Structural Health Management Using In Situ Acoustic Emission Monitoring International Journal of Fatigue 49 2013: pp. $81-89$. http://dx.doi.org/10.1016/j.ijfatigue.2012.12.001

4. He, Y.Z., Luo, F.L., Pan, M.C., Weng, F.B., Hu, X.C., Gao, J.Z., Liu,B. Pulsed Eddy Current Technique for 
Defect Detection in Aircraft Riveted Structures $N D T \& E$ International 43 (2) 2010: pp. 176-181.

5. Yuan, S.F., Qiu, L., Wang, Q., Miao, M., Yu, Z.H. Application Research of a Hybrid Piezoelectric-Optic Fiber Integrated Structural Acta Aeronautica et Astronautica Sinica 30 (2) 2009: pp. 348-356.

6. Guo, W.l., Shao, R.P., Feng, Q. A Review and Prospect of Damage Detection in Structures Journal of Vibration, Measurement \& Diagnosis 23 (2) 2003: pp. 79-85.

7. Cui, R.H., He, Y.T., Yu, Z.M., Li, H.P., Shu, W.J., Du, J.Q. Structural Surface Crack Monitoring Method Based on Electrical Potential Technique and Modern Surface Technology Chinese Journal of Mechanical Engineering 24 (4) 2011: pp. 601-606.

8. Hou, B., He, Y.T., Cui, R.H., Gao, C., Zhang, T. Crack monitoring method based on $\mathrm{Cu}$ coating sensor and electrical potential technique for metal structure Chinese Journal of Aeronautics 28 (3) 2015: pp. 932-938. http://dx.doi.org/10.1016/j.cja.2015.02.016

9. Liu, M.B., Li, B.B., Li, J.T., Lian, Y.Y. Smart Coating Sensor Applied in Crack Detection for Aircraft Applied Mechanics and Materials 330 2013: pp. 383-388.

10. Zhang, B., Wang, S., Li, X., Zhang, X., Yang, G., Qiu, M. Crack Width Monitoring of Concrete Structures Based on Smart Film Smart Materials and Structures 23(4) 2014: pp. $045031-045045$.
11. Takiuti, B.E., Júnior, V.L., Brennan, M.J., Arlindo, E.P.S., Orlandi, M.O. Damage Detection and Quantification Using Thin Film of ITO Nanocomposites Structural Health Monitoring, Volume 5: Proceedings of the 32 nd Imac, a Conference and Exposition on Structural Dynamics 2014. Springer Science \& Business 2014: pp. 207-213.

12. Nayeb-Hashemi, H., Swet, D., Vaziri, A. New Electrical Potential Method For Measuring Crack Growth in Nonconductive Materials Measurement 36 2004: pp. $121-129$.

13. Ashrafi, B., Johnson, L., Martinez-Rubi, Y., Martinez, M., Mrad, N. Single-Walled Carbon NanotubeModified Epoxy Thin Films for Continuous Crack Monitoring of Metallic Structures Structural Health Monitoring 11 (5) 2012: pp. 589-601.

14. Lidsky, L.M., Rothleder, S.D., Rose, D.J., Yoshikawa, S., Michelson, C., Mackin-Jr, R.J. Highly Ionized Hollow Cathode Discharge Journal of Applied Physics 33 (8) 1962: pp. $2490-2497$. http://dx.doi.org/10.1063/1.1729002

15. Hou, B., He, Y.T., Cui, R.H., Ding, H., Jiao, S.B. Fatigue Crack Monitoring of Metallic Structure Based on Coating Sensor Journal of Beijing University of Aeronautics and Astronautics 39 (10) 2013: pp.1298-1302. 